Nucl. Med. Biol. Vol. 19, No. 3, pp. 417-421, 1992

Int. J. Radiat. Appl. Instrum. Part B

Pergamon Press plc. Printed in Great Britain

$0883-2897 / 92 \$ 5.00+0.00$

\section{Streptavidin-biotinylated IgG Conjugates: a Simple Procedure for Reducing Polymer Formation}

\author{
RENATO B. DEL ROSARIO ${ }^{2}$, LEE ANN BARON', \\ RICHARD G. LAWTON ${ }^{1}$ and RICHARD L. WAHL ${ }^{2 *}$ \\ 'Department of Chemistry and ${ }^{2}$ Division of Nuclear \\ Medicine-Internal Medicine, University of Michigan \\ Medical Center, Ann Arbor, MI 48109-0028, U.S.A.
}

(Received 9 July 1991)

Disulfide links of the IgG2ak anti-ovarian carcinoma antibody, $5 \mathrm{G} 6.4$, were site-specifically biotinylated [ $\approx 2$ biotins/ IgG2a] using a novel crosslinking procedure using the biotin derivatized ETAC (equilibrium transfer alkylation crosslink reagent) 1a. Complexation of ETAC 1a biotinylated 5G6.4 on a column of immobilized protein $\mathrm{A}$ at high dilution, followed by passage of $\left[{ }^{125} \Gamma\right]$ streptavidin, washing and $\mathrm{pH}$ change leads to elution of a streptavidin-free product with a molecular mass in the $200-300 \mathrm{kDa}$ range. By contrast, direct mixing with $\left[{ }^{125} \mathrm{I}\right]$ streptavidin rapidly gave larger oligomers of $\geqslant 669$ and $\approx 440-669 \mathrm{kDa}$ molecular mass, respectively. The biodistribution of the $200-300 \mathrm{kDa}$ complex showed significantly diminished liver, kidney and spleen uptake as well as higher blood activity than the 440-669 kDa complex. The methodology represent the first application of ETAC chemistry to disulfide-bond directed biotinylation of antibodies and the synthesis of streptavidin antibody conjugates which minimizes their polymerization.

Developments in the use of biotinylated antibodies and pre-formed streptavidin biotinylated antibody complexes may have applicability to using such approaches in radioimmunoconjugate delivery (Hnatowich et al., 1987; Paganelli et al., 1990d; Pimm et al., 1988; Sinitsyn et al., 1989). Successful clinical studies have been reported by at least two groups (Rowlinson et al., 1988; Paganelli et al., $1990 a, b, c)$. Recently, we reported our findings concerning the potential applications of sulfhydryl site-directed biotinylation as a means of better preserving the immunoreactivity of biotinylated antibodies and their corresponding radiolabeled conjugates (del Rosario and Wahl, 1989, 1990). These earlier data grew from our initial observation that reduced interchain disulfide links of antibodies were readily mono-alkylated with an iodoacetamide derivative of biotin (del Rosario and Wahl, 1989). Following complexation with radio-streptavidin, the oligomeric conjugates retained in vivo immunological activity. However, we and others have recognized that proper control of molecular size and purification remains a key problem in the synthesis of homogeneous streptavidin-biotinylated-monoclonal IgG complexes for use in radioimmunoimaging (Hnatowich et al., 1987). Here

*Author for correspondence. we describe a simple method for minimizing the molecular size of the conjugate formed upon reaction with streptavidin which allows excess streptavidin to be easily separated from product without HPLC purification.

Biotinylation was accomplished using a novel procedure using the "ETAC" (equilibrium transfer alkylation) crosslink reagent 1a, prepared in our laboratories (del Rosario et al., 1989, 1990) (Fig. 1). In previous work (del Rosario et al., 1989, 1990; Liberatore et al., 1990) we have shown that ETAC reagents provide a unique site-specific pathway for attaching a wide range of bioprobes to antibodies, i.e. through insertion and formation of a stable three carbon crosslinked disulfide bridge (del Rosario et al., 1990). In contrast to our earlier papers, ETAC biotinylation proceeds through a bis-alkylation as opposed to a mono-alkylation (as in the reaction of haloacetyl derivatives) of disulfide bonds (del Rosario and Wahl, 1989, 1990).

We have recently reported the synthesis of the parent compound carboxy ETAC 1 and a biotin derivative, 1a (del Rosario et al., 1989). Biotinylated ETAC 1a (Fig. 1) was prepared by carbodiimide-mediated derivatization of carboxy-ETAC 1 with commercially available $5-(() N$ biotinoyl)aminohexanoyl)amino)pentylamine (Molecular Probes) (del Rosario et al., 1989). The anti-ovarian carcinoma IgG2ak antibody, 5G6.4 (Wahl et al., 1986) was chosen for the present study. 5G6.4 (produced from mouse ascites and purified by protein A chromatography) in $0.1 \mathrm{M}$ sodium phosphate $(\mathrm{pH} \approx 7)$ solution was reduced $\left(37^{\circ} \mathrm{C}\right.$, $5 \mathrm{~h}$ ) with a $\approx 150$ molar excess of DTT (Aldrich Chemical Co.). The mixture was treated with a DMSO solution of ETAC 1a (1:1 molar ratio with respect to DTT), vortexed and the emulsion allowed to react for $15-24 \mathrm{~h}$ at $37^{\circ} \mathrm{C}$. The reaction mixture was vortexed $(15 \mathrm{~min})$ and the clear supernatant desalted over Sephadex G-25 using $0.1 \mathrm{M}$ sodium phosphate buffer $(\mathrm{pH} \approx 7)$.

Site-specific crosslinking and biotinylation of interchain disulfide links of $5 \mathrm{G} 6.4$ was confirmed by reducing SDS-PAGE analysis (Pharmacia Phast System) of the crosslinked products. Since the new $-S-\{$ biotinoylETAC $-\mathrm{S}-$ chemical bonds were stable even at $100^{\circ} \mathrm{C}$ and not cleaved by reducing thiols, the crosslinked species appeared as new bands of $\approx \mathrm{H}_{2} \mathrm{~L}_{2}, \mathrm{H}_{2} \mathrm{~L}, \mathrm{H}_{2}$ and $\mathrm{HL}$ mass units in addition to uncrosslinked heavy $(\mathrm{H})$ and light $(\mathrm{L})$ chains on electrophoresis. These observations were identical to our previous findings (del Rosario et al., 1989, 1990; Laemmli, 1970; Liberatore et al., 1990). Laser densitometric measurement (Pharmacia Ultrascanner) of the Coomassie blue content on each band allowed calculation of an average of $\approx 2$ interchain $\mathrm{H}-\mathrm{H}$ and $\mathrm{H}-\mathrm{L}$ disulfide attached biotins per biotinylated 5G6.4 molecule (del Rosario et al., 1989, 1990). As expected the extended "spacer arm" of the new biotin derivative $1 \mathrm{a}$ allowed facile complexation with streptavidin as has been observed with derivatives of $N$-hydroxysuccinimide esters of biotin (Hnatowich et al., 1987). Reaction of biotinylated 5G6.4 (MW $\approx 150,000)$ with [ ${ }^{125}$ I]streptavidin ( $M W \approx 60,000$ ) in approximately equimolar ratios led to formation of complex oligomeric mixtures covering the $\approx 400,000$ to $\gg 669,000$ molecular mass region. The molecular weights of these conjugates were estimated from calibration plots of known standards using Pharmacia Superose 6 and 12 size-exclusion radio-FPLC as previously described (del Rosario and Wahl, 1990). Figure 2(a) shows a representative product profile. With excess biotinylated $5 \mathrm{G6} .4$, the predominant species was mass $\gg 669 \mathrm{kDa}$ (species A), whereas $\approx 2$ or more molar equivalents of streptavidin per mole of biotinylated $5 \mathrm{G} 6.4$ favored species in the lower $400-669 \mathrm{kDa}$ mass range (Species B) 


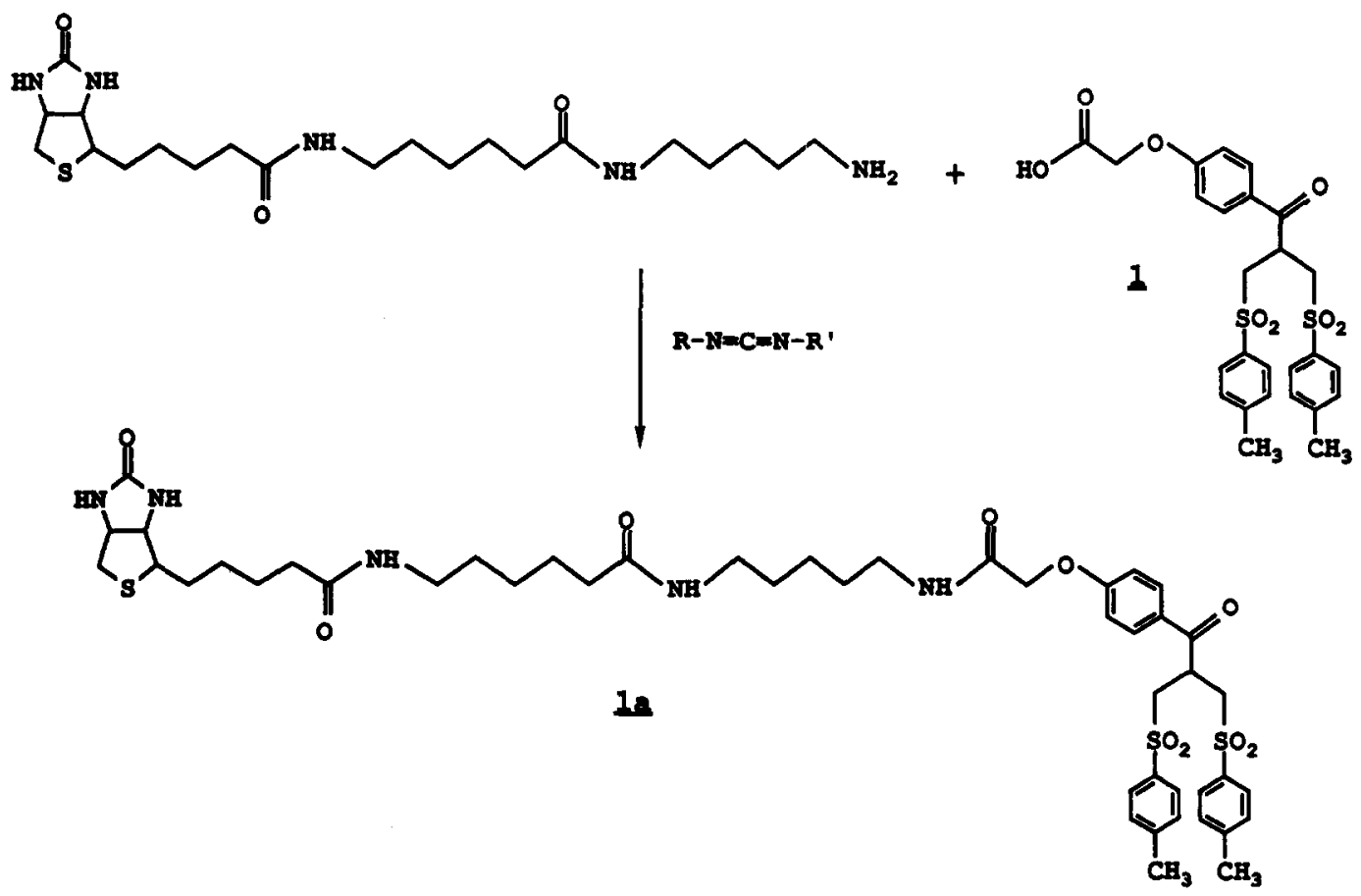

Fig. 1. Chemical structures of ETAC 1 and biotin derivative 1a.

Retention of immunoreactivity of the biotinylated and streptavidin-biotinylated 5G6.4 pre-formed complexes was verified by direct cell binding assays of the radiolabeled complex using human 77 IP3 target cells (del Rosario and Wahl, 1990). Direct binding of biotinylated 5G6.4 was indicated by specific binding $(60 \%)$ of $\left[{ }^{125} I\right]$ streptavidin on target cells pre-incubated with the biotinylated $5 \mathrm{G} 6.4$ conjugate. Preformed oligomeric species $A(\gg 669 \mathrm{kDa})$ or $B$ $(\approx 400->669 \mathrm{kDa}$ ) [Fig. 2(a)] both exhibited comparable or better $(60-80 \%)$ specific binding than [ $\left.{ }^{125} \mathrm{I}\right] 5 \mathrm{G} 6.4$ $(50-65 \%)$ labeled via the iodogen method (at substantial antigen excess). Flow cytometry results using fluorescent streptavidin were identical with those previously reported for the iodoacetyl-biotin conjugate of $5 \mathrm{G6.4}$ (del Rosario and Wahl, 1989, 1990). Species A [Fig. 2(b)] showed excellent stability in serum ( 5 days) as assessed by radio-FPLC. Similarly, complex formation was observed when ETAC. 1a biotinylated 5 G6.4 was allowed to $\mathrm{mix}$ with radiolabeled streptavidin in serum $(\approx 1 \mu \mathrm{g}$ biotinylated $5 G 6.4 / \mathrm{mL}$ serum) instead of buffer. The preservation of immunoreactivity and stability data are consistent with the hypothesis that ETAC conjugation occur at sites distant from the antigen binding sites and leads to relatively stable biotin ETAC 1a crosslinks in 5G6.4. However, a preliminary biodistribution study (mice, $n=3 /$ group) of product complex enriched in species $A$ ( $\gg 669 \mathrm{kDa}$ ) showed large liver $\left(0.263^{*} \pm 0.016\right)$ and spleen $(0.539 \pm 0.134)$ uptake with very little blood $(0.006 \pm 0.001)$ pool activity by $48 \mathrm{~h}$ in comparison to a $150 \mathrm{kDa}$ radiolabeled non-specific IgG2a antibody (UPC-10) [liver: $0.055 \pm 0.006$; spleen: $0.167 \pm 0.026$; blood: $0.111 \pm 0.066, P \leqslant 0.0005, P \leqslant 0.025$, $P \leqslant 0.10$, respectively. $\left.{ }^{*}\right]$ The above results closely parallel our previous studies concerning the effect of added antimouse antibody on the in vivo behavior of circulating intact radiolabeled UPC-10 and 5G6.4 (Wahl and Fisher, 1987) and implied that large complex size would present a substantial problem in targeting these antibody conjugates to tumors.

*All values are in $\% \mathrm{~kg} \mathrm{ID} / \mathrm{g}( \pm \mathrm{SEM})$.
To minimize formation of very large inter-antibody streptavidin oligomers (and their undesired non-target uptake in the liver and spleen), an alternative approach was devised using a protein affinity matrix. The strategy begins by first "anchoring" the same lot of biotinylated 5G6.4 on protein A (via its Fc portion) at $\mathrm{pH} 8-9$ (Ey et al., 1978). $\left[{ }^{125} \mathrm{I}\right]$ Radiostreptavidin was passed through the column briefly at high dilution to allow contact with the protein A-bound IgG. Unbound $\left[{ }^{125} \mathrm{I}\right] \mathrm{streptavidin}$ was then washed away and the 5G6.4-biotinylated streptavidin conjugate was saturated by passing a saturated buffer solution of biotin through the column to block available biotin binding sites on the streptavidin while still being bound to protein $A$. The product complex was conveniently cluted from off the column by washing at $\mathrm{pH} 5$.

A representative procedure is as follows. The above ETAC 1a-biotinylated 5G6.4 was thoroughly mixed with immobilized Protein A (Repligen ${ }^{\circledR}, \approx 200-300 \mu \mathrm{g} \mathrm{IgG2a/}$ $5 \mathrm{~mL}$ gel) suspended in $0.1 \mathrm{M}$ sodium phosphate buffer $(\mathrm{pH} \approx 8-9)$. The slurry was poured into a column. [25 $\mathrm{I}] \mathrm{Streptavidin}(30-40 \mu \mathrm{Ci} / \mu \mathrm{g}, 100-300 \mu \mathrm{Ci})$ was allowed to run through the column once. The column was washed several times with a saturated solution of biotin in $0.1 \mathrm{M}$ $(\mathrm{pH} \approx 8-9)$ sodium phosphate buffer until the eluent showed no change in background activity. Product complex was then eluted with $0.1 \mathrm{M}$ citrate buffer at $\mathrm{pH} 5(\approx 80 \mu \mathrm{Ci})$ and examined for free streptavidin by size-exclusion radioFPLC [Pharmacia Superose 6, Fig. 2(c)] analysis.

Products of significantly lower molecular weight $(\approx 200,000-300,000)$ with radio-FPLC profiles such as C [Fig. 2(c)] were eluted at $\mathrm{pH} 5$. The shoulder of peak $\mathrm{C}$ (higher molecular mass) in Fig. 2(c) was evident even when the radio-streptavidin used was partially blocked with $<4$ equivalents of biotin prior to contact with the immobilized biotinylated IgG. The biodistribution of species $\mathrm{C}$ was then compared with species $B$ in rats $(n=3)$ and is summarized in Fig. 3. The biodistribution (rats, $n=3 /$ group, $t=48 \mathrm{~h}$ ) of species $\mathrm{C}$ showed significantly diminished liver, kidney and spleen uptake as well as higher blood activity than the $440-669 \mathrm{kDa}$ complex (liver $\approx 2.4$ [ratio of $440-669 \mathrm{kDa}$ ) $200-300 \mathrm{kDa}$ activity], $P \leqslant 0.025$; kidney $\approx 2.7, P \leqslant 0.005$; 

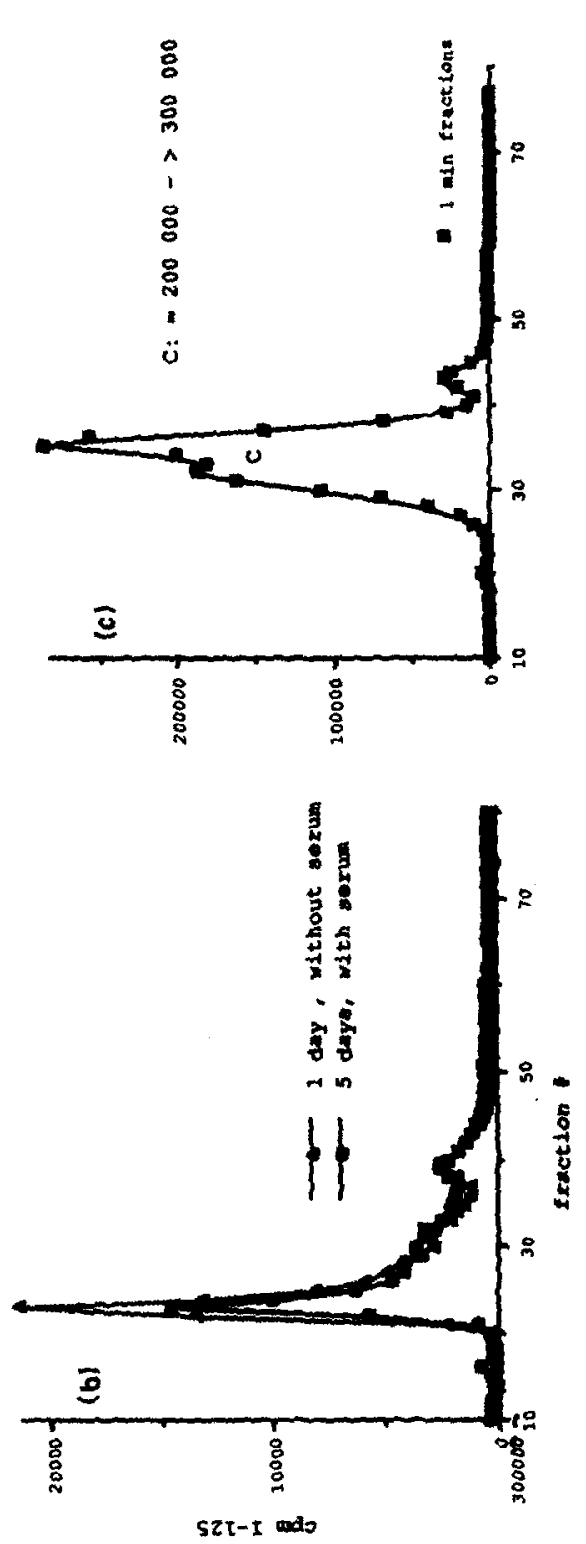

希

言

D。

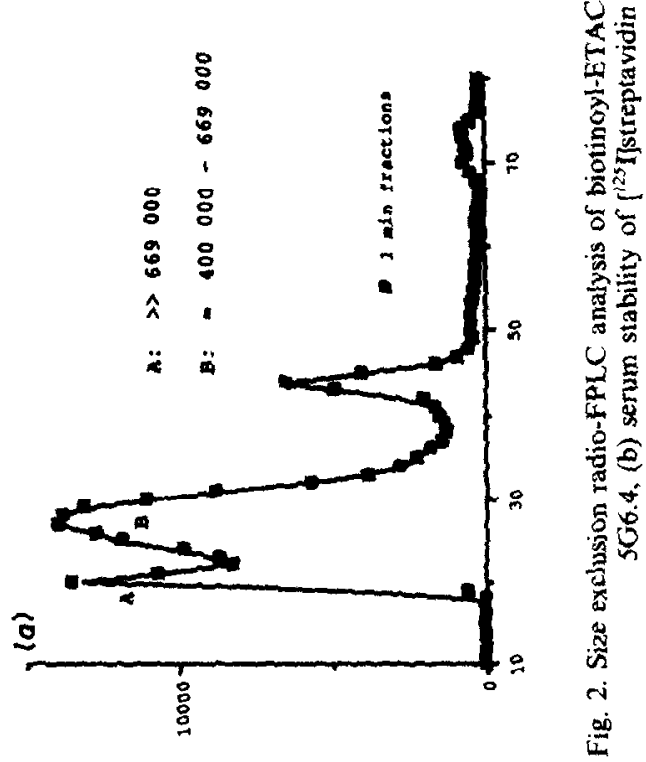




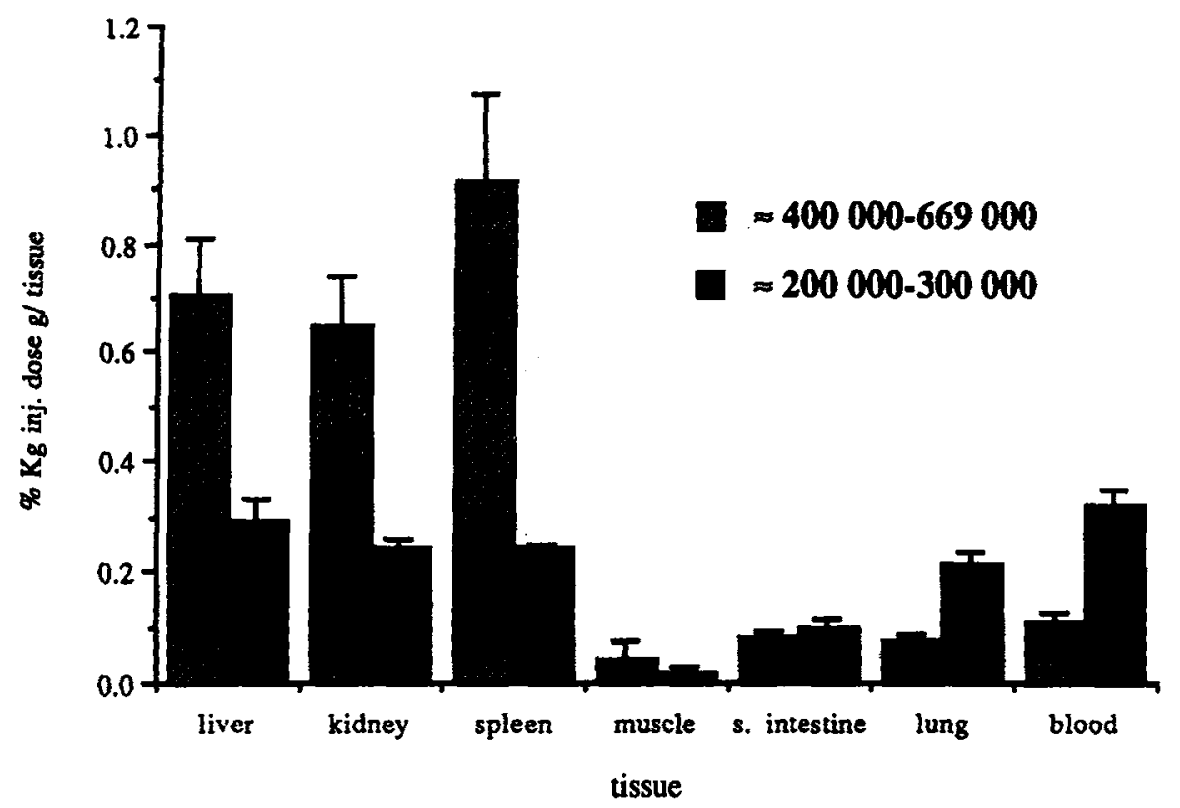

Fig. 3. Biodistribution of biotinylated 5G6.4 streptavidin complexes in rats $(n=3)$ as a function of size (species B: hatched; species C: solid).

spleen $\approx 3.8, \quad P \leqslant 0.01$; blood $[440-669 \mathrm{kDa} 200-$ $300 \mathrm{kDa}] \approx 0.35, P \leqslant 0.005\}$. Liver and spleen to blood ratios were decreased by $\approx 8 \times(P \leqslant 0.05)$ and $\approx 13 \times$ $(P \leqslant 0.05)$ for the $200 \mathrm{kDa}$ complex relative to the $440-669 \mathrm{kDa}$ product. The improved retention of the lower molecular weight complex in the blood appears due to avoiding high level delivery to the liver and spleen. Furthermore, this should result in better contact between the antibody and antigens present on tumor targets thus improving immunoconjugate targeting (Hnatowich et al., 1987).

In conclusion the above results provide (a) the first example of site-specitic biotinylation via a unique mechanism of concomitant crosslinking of reduced disulfide links, (b) further illustrates the relevance of immunoconjugate size in tissue localization, (c) demonstrates that improvements in biodistribution can clearly be achieved with a smaller complex and (d) illustrates a practical approach for avoiding high molecular weight oligomers. A limitation of the technique is that it may not be directly applicable to pre-targeting strategies if all streptavidin sites are preblocked by biotin. This can be addressed by using lesser quantities of biotin to allow only partial blocking (Green, 1975). The chemistry of the new biotin derivative, ETAC 1a further widens the scope and utility of immunologic applications of the biotin-streptavidin couple. Furthermore, the immobilization strategy described herein may find similar applicability in the synthesis of other antibody conjugates where polymer formation is undesirable (Alam et al., 1989).

Acknowledgements - We wish to thank Anaira Clavo, Susan J. Fisher, Gayle Ann Jackson and Dr Patricia J. Scott for providing tissue culture services, support animal studies and additional HPLC data. This research was funded in part by NIH Grants RO1 CA41531-02 and PO1 CA42768-01A1.

\section{References}

Alam F., Barth R. F. and Soloway A. H. (1989) Boron containing immunoconjugates for neutron capture therapy of cancer and for immunocytochemistry. Antibody Immunoconj. Radiopharm. 2, 145.
Ey P., Prowse S. and Jenkins C. (1978) Isolation of pure IgG1, IgG2a and lgG2b immunoglobulins from mouse serum using protein A sepharose. Immunochemistry 15, 429.

Green N. M. (1975) Avidin. Adv, Protein Chem. 29, 85. Hnatowich D. J., Virzi F. and Rusckowski M. (1987) Investigations of avidin and biotin for imaging applications. J. Nucl. Med. 28, 1294.

Laemmli V. K. (1970) Cleavage of structural proteins during the assembly of the head of bacteriophage T4. Nature 222, 680 .

Liberatore F. A., Comeau R. D., McKearin J. M., Pearson D. A., Belonga III B. Q., Brocchini S. J., Kath J., Philipps T., Oswell K. and Lawton T. G. (1990) Site-directed chemical modification and cross-linking of a monoclonal antibody using equilibrium transfer alkylating cross-link reagent. Bioconj. Chem. 1, 36.

Paganelli G., Magnani P., Rossetti C., Zito F., Belloni C., Pasini A., Sassi I., Sanvito F., Siccardi A. G. and Fazio F. (1990a) Tumor targeting in patients with ovarin cancer using biotinylated monoclonal antibodies and radioactive streptavidin. J. Nucl. Med. 31, 735 .

Paganelli G., Magnani P., Zito F., Villa E., Stella M., Lopalco L., Siccardi A. G. and Fazio F. (1990b) Antibody guided tumor detection in CEA positive patients using the avidin-biotin system. $J$, Nucl. Med. 31, 735.

Paganelli G., De Nardi P., Magnani P., Stella M., Pennacchioli L., Di Carlo V., Fazio F. and Siccardi A. G. (1990c) Antibody guided surgery in cancer patients: rapid blood clearance using I-124 labeled biotinylated monoclonal antibodies and cold avidin. J. Nucl. Med. 31, 853.

Paganelli G., Pervez S., Siccardi A. G., Rowlinson G., Deleidi G., Chiolerio F., Malcovati M., Scassellati G. A. and Epenetos A. A. (1990d) Intraperitoneal radio-localization of tumors pre-targeted by biotinylated monoclonal antibodies. Int. J. Cancer 45, 1184.

Pimm M. V., Fclls H. F., Pcrkins A. C. and Baldwin R. W. (1988) Iodine-131 and indium- 111 labeled avidin and streptavidin for pretargeted immunoscintigraphy with biotinylated antitimor monoclonal antibody. Nucl. $\mathrm{Med}$. Commun. 9, 931. 
del Rosario R. B. and Wahl R. L. (1989) Site-specific radiolabeling of monoclonal antibodies with biotin streptavidin. Nucl. Med. Biol. 16, 525.

del Rosario R. B. and Wahl R. L. (1990) Disulfide bondtargeted radiolabeling: tumor specificity of a streptavidinbiotinylated monoclonal antibody complex. Cancer Res. Suppl. 50, 804s.

del Rosario R. B., Wahl R. L., Brocchini S. J., Lawton R. G. and Smith R. H. (1990) Sulfhydryl site-specific cross-linking and labeling of monoclonal antibodies by a fluorescent equilibrium transfer crosslink reagent. Bioconj. Chem. 1, 51.

del Rosario R. B., Brocchini S. J., Baron L. A., Lawton R. G., Smith R. H., Jackson G. A. Scott P. A., Clavo A. C., Fisher S. J. and Wahl R. L. (1989) Sulfhydryl site-specific radiolabeling of monoclonal antibodies using "equilibrium transfer alkylation crosslink (ETAC)" reagents. Applications to the biotin streptavidin methodology. J. Nucl. Med. 30, 832.
Rowlinson G., Ruskowski M., Gionet M., Siebecker D., Snook D., Epenetos A. and Hnatowich D. J. (1988) Animal tumor localization studies with streptavidinconjugated antibody and labeled biotin. J. Nucl. Med. 29, 762.

Sinitsyn V. V., Mamontova A. G., Checkneva Y. Y., Shuyra A. A. and Domagatsky S. P. (1989) Rapid blood clearance of biotinylated IgG after infusion of avidin. J. Nucl. $\mathrm{Med}$. 30, 66.

Wahl R. L. and Fisher S. J. (1987) Intraperitoneal delivery of monoclonal antibodies: enhanced regional delivery advantage using intraveneous unlabeled anti-mouse antibody. Nucl. Med. Biol. 14, 611 .

Wahl R. L., Liebert M. L., Biesman B., Roberts J., Jackson G. A., Kronberg S. and Laino L. (1986) Production and characterization of a murine monoclonal antibody reactive with ovarian and other epithelial carcinomas. Proc. AACR 27, 355. 